\title{
High birth weight and large-for-gestational-age in singletons born after frozen compared to fresh embryo transfer, by gesta- tional week: a Nordic register study from the CoNARTaS group
}

Terho et al.

Large-for-gestational-age after frozen embryo transfer

\author{
A.M. Terho ${ }^{1}{ }^{*}$, S. Pelkonen ${ }^{1}$, S. OpdahI ${ }^{2}$, L.B. Romundstad ${ }^{3}{ }^{4}$, C. Bergh ${ }^{5}$, U.B. Wennerholm ${ }^{5}$, \\ A.A. Henningsen ${ }^{6}$, A. Pinborg 6 , M. Gissler ${ }^{7}$, and A. Tiitinen ${ }^{9}$ [AQ1] \\ 1. Department of Obstetrics and Gynaecology, Oulu University Hospital and University of Oulu, Oulu, Finland \\ 2. Department of Public Health and Nursing, Norwegian University of Science and Technology, Trondheim, Norway \\ 3. Center for Fertility and Health, Norwegian Institute of Public Health, Oslo, Norway \\ 4. Spiren Fertility Clinic, Trondheim, Norway \\ 5. Department of Obstetrics and Gynaecology, Institute of Clinical Sciences, Sahlgrenska Academy, University of Gothenburg, Sahl- \\ grenska University Hospital, Gothenburg, Sweden \\ 6. The Fertility Clinic, Copenhagen University Hospital, Rigshospitalet, Copenhagen, Denmark \\ 7. THL, Finnish Institute for Health and Welfare, Statistics and Registers Unit, Helsinki, Finland \\ 8. Department of Neurobiology, Care Sciences and Society, Karolinska Institutet, Stockholm, Sweden \\ 9. Department of Obstetrics and Gynaecology, Helsinki University Hospital and University of Helsinki, Helsinki, Finland [AQ2] [AQ3]
}

*Correspondence address. Department of Obstetrics and Gynaecology, Oulu University Hospital, PL 23, 90029 OYS, Oulu, Finland. E-mail: anna.terho@fimnet.fi

\section{ABSTRACT}

When do the differences in birth weights become apparent between singletons born after frozen embryo transfer (FET) and fresh embryo transfer (fresh ET)?

Mean birth weights after FET become significantly higher starting from gestational week (GW) 33 among boys and from GW 34 among girls.

In recent years, there has been a steep rise in recorded FET treatments, enabling widespread use of elective single embryo transfer, thus reducing the risks associated with multiple gestations. However, singletons born after FET are heavier and there is a higher risk of large-for-gestational-age (LGA) (birth weight $>90$ percentiles) compared to fresh ET. In contrast, risk of small-forgestational-age (SGA, birth weight $<10$ percentiles) is lower in singletons born after FET compared to fresh ET. The reasons, timing and consequences of these differences remain largely unclear. There is limited evidence about whether this difference in growth develops before the last trimester of pregnancy.

This retrospective Nordic register-based cohort study compared singletons born after FET ( $n=17500)$ to singletons born after fresh ET $(n=69510)$ and natural conception (NC, $n=3311588$ ). All live born singletons born between the years 2000 and 2015 in Denmark, Norway and Sweden at gestational age $\geq 22$ weeks were included from the population-based Committee of Nordic ART and Safety (CoNARTaS) study population.

Children born after FET were compared to those born after fresh ET and NC for mean birth weight and proportion of LGA and SGA for each GW at birth. Chi-square test and tests for relative proportions were used to compare categorical variables and Student's $t$-test was used to compare continuous variables. Adjusted odds ratios (aORs) for LGA and SGA were calculated using logistic regressions, adjusting for year of birth, maternal age, parity, BMI, chronic hypertension, diabetes, smoking and offspring sex. 
Mean birth weights were significantly higher after FET compared to fresh ET starting from GW 33 (range from $75 \mathrm{~g}$ to $228 \mathrm{~g}$ by week) for boys and starting from GW 34 (range from $90 \mathrm{~g}$ to $236 \mathrm{~g}$ by week) for girls. Boys born after FET had a significantly higher proportion of LGA (11.0-15.1\%) at birth between GW 36 and 42, compared to those born after fresh ET (7.1-9.4\%) (range from $P$ $<0.001$ to $P=0.048$ by week). For girls born after FET, the difference was seen between GW 37 and 42 (10.6-13.4\%) compared to those born after fresh ET (6.6-8.0\%) (range from $P<0.001$ to $P=0.009$ by week). The proportion of SGA was significantly lower among boys born after FET (7.6-8.7\%) compared to fresh ET (11.9-13.6\%) between GW 36 and 42 (range from $P<0.001$ to $P=$ 0.016 by week). For girls born after FET, the difference was seen between GW 38 and 42 (7.0-9.3\%) compared to those born after fresh ET (13.0-14.6\%) $(P<0.001)$. The proportion of LGA $(12.3-15.1 \%)$ was significantly higher for boys born after FET between GW 38 and $41(P<0.001)$ and for girls born after FET $(12.6-13.4 \%)$ between GW 37 and 40 (range from $P<0.001$ to $P=0.018$ by week), compared to naturally conceived boys (9.7-9.9\%) and girls (9.0-10.0\%). All singletons born after FET had a higher risk of LGA compared to singletons born after fresh ET (aOR 1.87, 95\% Cl 1.76-1.98) and singletons born after NC (aOR $1.28,95 \% \mathrm{Cl}$ 1.22-1.35).

There may be residual confounding factors that we were not able to control for, most importantly the causes of preterm birth, which may also influence foetal growth. A further limitation is that we have no knowledge on growth patterns between implantation and GW 22. Finally, the number of children born extremely preterm or post-term was limited even in this large study population.

This is, to date, the largest study on birth weights among preterm and term ART singletons with a population-based design and NC control group. The results suggest that the freeze-thaw process is associated with higher birthweights and greater risk of LGA at least in the last trimester of pregnancy. This is an important aspect of the safety profile of ART. More research is needed on the long-term outcome of these children.

The CoNARTaS collaboration has received the following funding: the Nordic Trial Alliance: a pilot project jointly funded by the Nordic Council of Ministers and NordForsk [71450], the Central Norway Regional Health Authorities [46045000], the Norwegian Cancer Society [182356-2016], the Nordic Federation of Obstetrics and Gynaecology [NF13041, NF15058, NF16026 and NF17043], the Interreg Öresund-Kattegat-Skagerrak European Regional Development Fund (ReproUnion project) and the Research Council of Norway's Centre of Excellence funding scheme [262700]. None of the authors have any competing interests to declare.

ISRCTN11780826.

Keywords: assisted reproduction / frozen embryo transfer / FET /large-for-gestational-age / LGA

\section{FUNDING}

Nordic Council of Ministers and NordForsk71450Central Norway Regional Health Authorities46045000Norwegian Cancer Society182356-2016Nordic Federation of Obstetrics and GynaecologyNF13041NF15058NF16026 NF17043Interreg Öresund-KattegatSkagerrak European Regional Development Fund10.13039/501100008530Research Council of Norway's Centre of Excellence262700Nordic Council of Ministers and NordForsk71450Central Norway Regional Health Authorities46045000Norwegian Cancer Society182356-2016Nordic Federation of Obstetrics and GynaecologyNF13041NF15058NF16026 NF17043Interreg Öresund-Kattegat-Skagerrak European Regional Development Fund10.13039/501100008530Research Council of Norway's Centre of Excellence262700

\section{Introduction}

Freezing of human embryos with thawing and transfer in a subsequent cycle (frozen embryo transfer, FET) is a wellestablished part of ART. In recent years, there has been a steep rise in recorded FET treatments, and the relative proportion of FET to fresh embryo transfer (fresh ET) was 40.3\% in Europe in 2015 (De Geyter et al., 2020). FET practice enables the widespread use of elective single embryo transfer (eSET), thus reducing the risks associated with multiple gestations (Thurin et al., 2004; Tiitinen and Gissler, 2004; Henningsen et al., 2015). Still, safety monitoring of different ART modalities is of utmost importance, since already one in 50 children born in Europe is the result of ART treatment (De Geyter et al., 2020).

It is well known that singletons born after FET have a higher risk of being born large-for-gestational-age (LGA) than singletons born after fresh ET. This has been shown in a number of large register-based cohort studies (Pelkonen et al., 2010; Sazonova et al., 2012; Wennerholm et al., 2013; Pinborg et al., 2014) and meta-analyses (Maheshwari et 
al., 2018; Sha et al., 2018). In contrast, singletons born after fresh ET have been shown to have a higher risk of smallfor-gestational-age (SGA) compared to FET (Pelkonen et al., 2010; Wennerholm et al., 2013; Pinborg et al., 2014; Maheshwari et al., 2018). Both small and large birth weights may be predictors of obesity and cardiometabolic risk factors and disease in adulthood (Barker, 2007; Taal et al., 2013; Kapral et al., 2018).

There is limited knowledge on the foetal growth patterns during pregnancies following different ART modalities. There is some evidence that growth differences develop before the last trimester of pregnancy (Li et al., 2014; Ginod et al., 2018). In this study, we aimed to investigate when this difference in birth weights occurs between singletons born after FET compared to singletons born after fresh ET and natural conception (NC). Thus, we estimated mean birth weights and proportions of SGA, appropriate-for-gestational-age (AGA) and LGA according to gestational age and sex.

\section{Materials and methods}

\section{Study population}

The CoNARTaS study population consists of all ART and NC pregnancies from Denmark, Finland, Norway and Sweden. Data were obtained from national ART registers and Medical Birth Registers and linked at the individual level using the national identity number assigned to all residents in each Nordic country at birth or immigration (Opdahl et al., 2019). For this study, data from Denmark, Norway and Sweden were used, but data on different ART modalities were not available from Finland. Live-born singletons (gestational age $\geq 22$ weeks at birth) born between the years 2000 and 2015 were included, $n=17500$ for FET, $n=69510$ for fresh ET and $n=3311588$ for NC. Children conceived after ovulation induction and intrauterine insemination (IUI) are included in the NC group in this study. In both FET and fresh ET groups, some oocyte donation pregnancies were included (1.8-2.4\%).

Gestational age for ART pregnancies was based on the number of culture days and the date of embryo transfer, and that for NC pregnancies was based on ultrasound (US) examination or last menstrual period if information on US was missing. Gestational weeks (GWs) used in this study are completed weeks. All analyses were performed using $<10$ percentiles and $>90$ percentiles according to the CoNARTaS data, as cut-off levels for SGA and LGA, respectively. We excluded cases with birthweight $<300 \mathrm{~g}$ or $\geq 6000 \mathrm{~g}$ and birthweight for gestational age $\leq-6$ and $\geq+6$ SDs (Marsal et al., 1996).

As regards to background factors, we had access to data on country, ART characteristics, embryo culture duration, number of embryos transferred, year of birth, maternal age, parity, prepregnancy BMI, smoking in pregnancy, level of education, diabetes, chronic hypertension, hypertensive disorders of pregnancy, induction of labour and caesarean section, as well as paternal age (Table I).

Table I Characteristics of pregnancies originated after frozen embryo transfer (FET), fresh embryo transfer (fresh ET) and natural conception (NC) categorized by birth weight into small-for-gestational-age (SGA, $<10$ percentiles), appropriate-for-gestationalage (AGA) and large-for-gestational-age (LGA, >90 percentiles). [AQ6]

\begin{tabular}{|c|c|c|c|c|c|c|c|c|c|c|}
\hline & \multicolumn{3}{|c|}{ FET $(n=17500)$} & \multicolumn{3}{|c|}{ Fresh ET $(n=69510)$} & \multicolumn{3}{|c|}{$\mathrm{NC}(\mathrm{n}=3311588)$} & \multirow{2}{*}{$\begin{array}{l}P \text {-val- } \\
\text { ue, }{ }^{\text {a }} \text { FET } \\
\text { vs fresh } \\
\text { ET }\end{array}$} \\
\hline & $\begin{array}{l}\text { SGA (n } \\
=1445)\end{array}$ & $\begin{array}{l}\text { AGA (n } \\
=13835)\end{array}$ & $\begin{array}{l}\text { LGA (n } \\
=2220)\end{array}$ & $\begin{array}{l}\text { SGA (n } \\
=9135)\end{array}$ & $\begin{array}{l}\text { AGA (n } \\
=55251)\end{array}$ & $\begin{array}{l}\text { LGA (n } \\
=5124)\end{array}$ & $\begin{array}{l}\text { SGA }(n= \\
326911)\end{array}$ & $\begin{array}{l}\text { AGA }(n= \\
2660928)\end{array}$ & $\begin{array}{l}\text { LGA }(\mathrm{n}= \\
323749)\end{array}$ & \\
\hline \multicolumn{11}{|l|}{$\begin{array}{l}\text { Country, n } \\
(\%)\end{array}$} \\
\hline Denmark & $\begin{array}{l}287 \\
(9.0)\end{array}$ & $\begin{array}{l}2530 \\
(78.9)\end{array}$ & $\begin{array}{l}388 \\
(12.1)\end{array}$ & $\begin{array}{l}3165 \\
(13.8)\end{array}$ & $\begin{array}{l}18151 \\
(78.9)\end{array}$ & $\begin{array}{l}1692 \\
(7.4)\end{array}$ & $\begin{array}{l}93175 \\
(10.8)\end{array}$ & $\begin{array}{l}686965 \\
(79.8)\end{array}$ & $\begin{array}{l}80241 \\
(9.3)\end{array}$ & \\
\hline Norway & $\begin{array}{l}240 \\
(7.1)\end{array}$ & $\begin{array}{l}2664 \\
(79.4)\end{array}$ & $\begin{array}{l}453 \\
(13.5)\end{array}$ & $\begin{array}{l}1944 \\
(12.9)\end{array}$ & $\begin{array}{l}12049 \\
(79.9)\end{array}$ & $\begin{array}{l}1091 \\
(7.2)\end{array}$ & $\begin{array}{l}85610 \\
(9.6)\end{array}$ & $\begin{array}{l}713903 \\
(80.5)\end{array}$ & \begin{tabular}{|l|}
87796 \\
$(9.9)$
\end{tabular} & \\
\hline Sweden & $\begin{array}{l}918 \\
(8.4)\end{array}$ & $\begin{array}{l}8641 \\
(79.0)\end{array}$ & $\begin{array}{l}1379 \\
(12.6)\end{array}$ & $\begin{array}{l}4026 \\
(12.8)\end{array}$ & $\begin{array}{l}25051 \\
(79.7)\end{array}$ & $\begin{array}{l}2341 \\
(7.5)\end{array}$ & $\begin{array}{l}148126 \\
(9.5)\end{array}$ & $\begin{array}{l}1260060 \\
(80.6)\end{array}$ & $\begin{array}{l}155712 \\
(10.0)\end{array}$ & \\
\hline
\end{tabular}




\begin{tabular}{|c|c|c|c|c|c|c|c|c|c|c|}
\hline & \multicolumn{3}{|c|}{ FET $(n=17500)$} & \multicolumn{3}{|c|}{ Fresh ET $(\mathrm{n}=69510)$} & \multicolumn{3}{|c|}{$\mathrm{NC}(\mathrm{n}=3311588)$} & \multirow{2}{*}{$\begin{array}{l}P \text {-val- } \\
\text { ue, }{ }^{\text {a }} \text { FET } \\
\text { vs fresh } \\
\text { ET }\end{array}$} \\
\hline & $\begin{array}{l}\text { SGA }(\mathrm{n} \\
=1445)\end{array}$ & $\begin{array}{l}\text { AGA }(\mathrm{n} \\
=13835)\end{array}$ & $\begin{array}{l}\text { LGA }(\mathrm{n} \\
=2220)\end{array}$ & $\begin{array}{l}\text { SGA }(\mathrm{n} \\
=9135)\end{array}$ & $\begin{array}{l}\text { AGA }(\mathrm{n} \\
=55251)\end{array}$ & $\begin{array}{l}\text { LGA }(\mathrm{n} \\
=5124)\end{array}$ & $\begin{array}{l}\text { SGA }(\mathrm{n}= \\
326911)\end{array}$ & $\begin{array}{l}\mathrm{AGA}(\mathrm{n}= \\
2660928)\end{array}$ & $\begin{array}{l}\text { LGA }(\mathrm{n}= \\
323749)\end{array}$ & \\
\hline $\begin{array}{l}\text { Maternal } \\
\text { age, mean } \\
\text { (SD) }\end{array}$ & $\begin{array}{l}34.7 \\
(4.3)\end{array}$ & $34.9(4.2)$ & $\begin{array}{l}35.1 \\
(4.2)\end{array}$ & $\begin{array}{l}34.3 \\
(4.3)\end{array}$ & $34.4(4.3)$ & $\begin{array}{l}34.7 \\
(4.3)\end{array}$ & $30.4(5.3)$ & $30.9(5.1)$ & $31.7(4.9)$ & $<0.001$ \\
\hline $\begin{array}{l}\text { Maternal } \\
\text { BMI, mean } \\
\text { (SD) }\end{array}$ & $\begin{array}{l}23.6 \\
(3.9)\end{array}$ & $24.1(4)$ & $\begin{array}{l}25.7 \\
(4.4)\end{array}$ & $\begin{array}{l}23.7 \\
(4.0)\end{array}$ & $24.2(4)$ & $\begin{array}{l}26.1 \\
(4.6)\end{array}$ & 23.7 (4.6) & $24.4(4.6)$ & $26.5(5.4)$ & 0.717 \\
\hline $\begin{array}{l}\text { Missing, } \\
\text { n (\%) }\end{array}$ & $\begin{array}{l}205 \\
(14.2)\end{array}$ & $\begin{array}{l}2439 \\
(17.6)\end{array}$ & $\begin{array}{l}437 \\
(19.7)\end{array}$ & $\begin{array}{l}2283 \\
(25.0)\end{array}$ & $\begin{array}{l}14455 \\
(26.2)\end{array}$ & $\begin{array}{l}1463 \\
(28.6)\end{array}$ & $\begin{array}{l}103964 \\
(31.8)\end{array}$ & $\begin{array}{l}837681 \\
(31.5)\end{array}$ & $\begin{array}{l}107770 \\
(33.3)\end{array}$ & \\
\hline $\begin{array}{l}\text { Primipar- } \\
\text { ous, n (\%) }\end{array}$ & $\begin{array}{l}1023 \\
(70.8)\end{array}$ & $\begin{array}{l}7673 \\
(55.4)\end{array}$ & $\begin{array}{l}922 \\
(41.5)\end{array}$ & $\begin{array}{l}7314 \\
(80.0)\end{array}$ & $\begin{array}{l}38346 \\
(69.4)\end{array}$ & $\begin{array}{l}2902 \\
(56.6)\end{array}$ & $\begin{array}{l}189755 \\
(58.0)\end{array}$ & $\begin{array}{l}1144545 \\
(43)\end{array}$ & $\begin{array}{l}87069 \\
(26.9)\end{array}$ & $<0.001$ \\
\hline Missing & $5(0.3)$ & $25(0.2)$ & $<5(0.1)$ & $60(0.7)$ & $326(0.6)$ & $32(0.6)$ & $1599(0.5)$ & $8865(0.3)$ & $784(0.2)$ & \\
\hline $\begin{array}{l}\text { Maternal } \\
\text { smoking, n } \\
(\%)\end{array}$ & $\begin{array}{l}100 \\
(6.9)\end{array}$ & $617(4.5)$ & $81(3.6)$ & $\begin{array}{l}993 \\
(10.9)\end{array}$ & $\begin{array}{l}3890 \\
(7.0)\end{array}$ & $\begin{array}{l}309 \\
(6.0)\end{array}$ & $\begin{array}{l}70413 \\
(21.5)\end{array}$ & $\begin{array}{l}361940 \\
(13.6)\end{array}$ & \begin{tabular}{|l}
32378 \\
$(10.0)$
\end{tabular} & $<0.001$ \\
\hline Missing & $48(3.3)$ & $467(3.4)$ & $81(3.6)$ & $\begin{array}{l}288 \\
(3.2)\end{array}$ & $\begin{array}{l}1805 \\
(3.3)\end{array}$ & $\begin{array}{l}162 \\
(3.2)\end{array}$ & $9825(3)$ & $82606(3)$ & $\begin{array}{l}10964 \\
(3.4)\end{array}$ & \\
\hline \multicolumn{11}{|l|}{$\begin{array}{l}\text { Maternal } \\
\text { education, }{ }^{\text {b }} \\
\text { n (\%) }\end{array}$} \\
\hline \begin{tabular}{|l|}
\multicolumn{1}{|c|}{ Basic/ } \\
upper sec- \\
ondary
\end{tabular} & $\begin{array}{l}333 \\
(23)\end{array}$ & $\begin{array}{l}3106 \\
(22.4)\end{array}$ & \begin{tabular}{|l|}
527 \\
$(23.7)$
\end{tabular} & $\begin{array}{l}1924 \\
(21.1)\end{array}$ & $\begin{array}{l}11715 \\
(21.2)\end{array}$ & $\begin{array}{l}1171 \\
(22.8)\end{array}$ & $\begin{array}{l}95871 \\
(29.3)\end{array}$ & $\begin{array}{l}749394 \\
(28.2)\end{array}$ & $\begin{array}{l}95486 \\
(29.5)\end{array}$ & $<0.001$ \\
\hline al Vocation- & $\begin{array}{l}222 \\
(15.4)\end{array}$ & $\begin{array}{l}1975 \\
(14.3)\end{array}$ & $\begin{array}{l}272 \\
(12.3)\end{array}$ & $\begin{array}{l}1711 \\
(18.7)\end{array}$ & $\begin{array}{l}9860 \\
(17.8)\end{array}$ & $\begin{array}{l}955 \\
(18.6)\end{array}$ & $\begin{array}{l}46014 \\
(14.1)\end{array}$ & $\begin{array}{l}376890 \\
(14.2)\end{array}$ & $\begin{array}{l}47955 \\
(14.8)\end{array}$ & $<0.001$ \\
\hline ty & $\begin{array}{l}564 \\
(39)\end{array}$ & $5521(40)$ & $\begin{array}{l}898 \\
(40.5)\end{array}$ & $\begin{array}{l}3159 \\
(34.6)\end{array}$ & $\begin{array}{l}19814 \\
(35.9)\end{array}$ & $\begin{array}{l}1780 \\
(34.7)\end{array}$ & $\begin{array}{l}70445 \\
(21.5)\end{array}$ & $\begin{array}{l}661819 \\
(24.9)\end{array}$ & $\begin{array}{l}79473 \\
(24.5)\end{array}$ & $<0.001$ \\
\hline Missing & $\begin{array}{l}326 \\
(22.6)\end{array}$ & $\begin{array}{l}3233 \\
(23.4)\end{array}$ & \begin{tabular}{|l|}
523 \\
$(23.6)$
\end{tabular} & $\begin{array}{l}2341 \\
(25.6)\end{array}$ & $\begin{array}{l}13862 \\
(25.1)\end{array}$ & $\begin{array}{l}1218 \\
(23.8)\end{array}$ & $\begin{array}{l}114581 \\
(35)\end{array}$ & $\begin{array}{l}872825 \\
(32.8)\end{array}$ & $\begin{array}{l}100835 \\
(31.1)\end{array}$ & \\
\hline $\begin{array}{l}\text { Paternal } \\
\text { age, }{ }^{\mathrm{c}} \text { mean } \\
\text { (SD) }\end{array}$ & $\begin{array}{l}37.2 \\
(5.6)\end{array}$ & $37.2(5.7)$ & $\begin{array}{l}37.3 \\
(5.6)\end{array}$ & $\begin{array}{l}36.6 \\
(5.7)\end{array}$ & $36.5(5.7)$ & $\begin{array}{l}36.7 \\
(5.8)\end{array}$ & $33(6.5)$ & $33.1(6.2)$ & $33.7(6)$ & $<0.001$ \\
\hline $\begin{array}{l}\text { Missing, } \\
\mathrm{n}(\%)\end{array}$ & $\begin{array}{l}300 \\
(20.8)\end{array}$ & $\begin{array}{l}2711 \\
(19.6)\end{array}$ & $\begin{array}{l}421 \\
(19)\end{array}$ & $\begin{array}{l}3228 \\
(35.3)\end{array}$ & $\begin{array}{l}18461 \\
(33.4)\end{array}$ & $\begin{array}{l}1715 \\
(33.5)\end{array}$ & $\begin{array}{l}97658 \\
(29.9)\end{array}$ & $\begin{array}{l}707784 \\
(26.6)\end{array}$ & $\begin{array}{l}81928 \\
(25.3)\end{array}$ & \\
\hline ICSI, n (\%) & $\begin{array}{l}534 \\
(37)\end{array}$ & $\begin{array}{l}4963 \\
(35.9)\end{array}$ & $\begin{array}{l}805 \\
(36.3)\end{array}$ & $\begin{array}{l}3669 \\
(40.2)\end{array}$ & $\begin{array}{l}23186 \\
(42)\end{array}$ & $\begin{array}{l}2223 \\
(43.4)\end{array}$ & NA & NA & NA & $<0.001$ \\
\hline Missing & $\begin{array}{l}113 \\
(7.8)\end{array}$ & $\begin{array}{l}1003 \\
(7.2)\end{array}$ & $\begin{array}{l}184 \\
(8.3)\end{array}$ & $16(0.2)$ & $128(0.2)$ & $15(0.3)$ & NA & NA & NA & \\
\hline \multicolumn{11}{|c|}{\begin{tabular}{|l|} 
Culture du- \\
ration, days, \\
n (\%)
\end{tabular}} \\
\hline $2-3$ & \begin{tabular}{|l|}
976 \\
$(67.5)$
\end{tabular} & \begin{tabular}{|l|}
8840 \\
$(63.9)$
\end{tabular} & $\begin{array}{l}1437 \\
(64.8)\end{array}$ & $\begin{array}{l}7383 \\
(80.9)\end{array}$ & $\begin{array}{l}43695 \\
(79.1)\end{array}$ & $\begin{array}{l}3220 \\
(77.1)\end{array}$ & NA & NA & NA & $<0.001$ \\
\hline$\geq 5$ & $\begin{array}{l}280 \\
(19.4)\end{array}$ & $\begin{array}{l}3083 \\
(22.2)\end{array}$ & $\begin{array}{l}489 \\
(21.9)\end{array}$ & $\begin{array}{l}522 \\
(5.7)\end{array}$ & $\begin{array}{l}3684 \\
(6.6)\end{array}$ & $362(7)$ & NA & NA & NA & $<0.001$ \\
\hline
\end{tabular}




\begin{tabular}{|c|c|c|c|c|c|c|c|c|c|c|}
\hline & \multicolumn{3}{|c|}{ FET $(\mathrm{n}=17500)$} & \multicolumn{3}{|c|}{ Fresh ET $(\mathrm{n}=69510)$} & \multicolumn{3}{|c|}{$\mathrm{NC}(\mathrm{n}=3311588)$} & \multirow{2}{*}{$\begin{array}{l}P \text {-val- } \\
\text { ue, }{ }^{\text {a }} \text { FET } \\
\text { vs fresh } \\
\text { ET }\end{array}$} \\
\hline & $\begin{array}{l}\text { SGA (n } \\
=1445)\end{array}$ & $\begin{array}{l}\text { AGA (n } \\
=13835)\end{array}$ & $\begin{array}{l}\text { LGA }(\mathrm{n} \\
=2220)\end{array}$ & $\begin{array}{l}\text { SGA }(n \\
=9135)\end{array}$ & $\begin{array}{l}\text { AGA (n } \\
=55251)\end{array}$ & $\begin{array}{l}\text { LGA }(\mathrm{n} \\
=5124)\end{array}$ & $\begin{array}{l}\text { SGA (n= } \\
326911)\end{array}$ & $\begin{array}{l}\text { AGA }(n= \\
2660928)\end{array}$ & $\begin{array}{l}\text { LGA }(\mathrm{n}= \\
323749)\end{array}$ & \\
\hline Missing & $\begin{array}{l}189 \\
(13.1)\end{array}$ & $\begin{array}{l}1912 \\
(13.8)\end{array}$ & $\begin{array}{l}294 \\
(13.2)\end{array}$ & $\begin{array}{l}1230 \\
(13.5)\end{array}$ & $\begin{array}{l}7872 \\
(14.2)\end{array}$ & $\begin{array}{l}814 \\
(15.9)\end{array}$ & NA & NA & NA & \\
\hline SET, n (\%) & $\begin{array}{l}1042 \\
(72.1)\end{array}$ & $\begin{array}{l}10023 \\
(72.4)\end{array}$ & $\begin{array}{l}1619 \\
(73)\end{array}$ & $\begin{array}{l}5073 \\
(55.5) \\
\end{array}$ & $\begin{array}{l}30257 \\
(54.7)\end{array}$ & $\begin{array}{l}2647 \\
(51.6)\end{array}$ & NA & NA & NA & $<0.001$ \\
\hline Missing & $24(1.7)$ & $308(2.2)$ & $53(2.4)$ & $\begin{array}{l}387 \\
(4.2)\end{array}$ & $\begin{array}{l}2453 \\
(4.4)\end{array}$ & $\begin{array}{l}238 \\
(4.6)\end{array}$ & NA & NA & NA & \\
\hline $\begin{array}{l}\text { Diabetes, }{ }^{\mathrm{d}} \mathrm{n} \\
(\%)\end{array}$ & $<5(0.2)$ & $39(0.3)$ & $41(1.8)$ & $12(0.1)$ & $135(0.2)$ & $62(1.2)$ & $351(0.1)$ & $5039(0.2)$ & 3771 (1.2) & $<0.001$ \\
\hline $\begin{array}{l}\text { Chronic hy- } \\
\text { pertension, } \\
\text { n }(\%)\end{array}$ & $25(1.7)$ & $115(0.8)$ & $19(0.9)$ & $\begin{array}{l}141 \\
(1.5)\end{array}$ & $490(0.9)$ & $57(1.1)$ & 3809 (1.2) & $17501(0.7)$ & $2354(0.7)$ & $<0.001$ \\
\hline HDP, n (\%) & $\begin{array}{l}175 \\
(12.1)\end{array}$ & $984(7.1)$ & $\begin{array}{l}150 \\
(6.8)\end{array}$ & $\begin{array}{l}910 \\
(10.0)\end{array}$ & $\begin{array}{l}2863 \\
(5.2)\end{array}$ & $\begin{array}{l}330 \\
(6.4)\end{array}$ & $\begin{array}{l}26680 \\
(8.2)\end{array}$ & $\begin{array}{l}104017 \\
(3.9)\end{array}$ & $\begin{array}{l}14082 \\
(4.3)\end{array}$ & $<0.001$ \\
\hline $\begin{array}{l}\text { Induction of } \\
\text { labour, n } \\
(\%)\end{array}$ & $\begin{array}{l}504 \\
(34.9)\end{array}$ & $\begin{array}{l}3364 \\
(24.3)\end{array}$ & $\begin{array}{l}578 \\
(26)\end{array}$ & $\begin{array}{l}2266 \\
(24.8)\end{array}$ & $\begin{array}{l}10270 \\
(18.6)\end{array}$ & $\begin{array}{l}1190 \\
(23.2)\end{array}$ & $\begin{array}{l}65035 \\
(19.9)\end{array}$ & $\begin{array}{l}366202 \\
(13.8)\end{array}$ & $\begin{array}{l}55822 \\
(17.2)\end{array}$ & $<0.001$ \\
\hline $\begin{array}{l}\text { Caesarean } \\
\text { section, } \mathrm{n} \\
(\%)\end{array}$ & $\begin{array}{l}423 \\
(29.3)\end{array}$ & $3731(27)$ & $\begin{array}{l}862 \\
(38.8)\end{array}$ & $\begin{array}{l}2425 \\
(26.5)\end{array}$ & $\begin{array}{l}13358 \\
(24.2)\end{array}$ & $\begin{array}{l}1902 \\
(37.1)\end{array}$ & $\begin{array}{l}61595 \\
(18.8)\end{array}$ & $\begin{array}{l}415118 \\
(15.6)\end{array}$ & $\begin{array}{l}76959 \\
(23.8)\end{array}$ & $<0.001$ \\
\hline $\begin{array}{l}\text { Preterm } \\
\text { births, n (\%) }\end{array}$ & $93(6.4)$ & 909 (6.6) & $\begin{array}{l}130 \\
(5.9)\end{array}$ & $\begin{array}{l}686 \\
(7.5)\end{array}$ & $\begin{array}{l}4341 \\
(7.9)\end{array}$ & $\begin{array}{l}428 \\
(8.4)\end{array}$ & $\begin{array}{l}15829 \\
(4.8)\end{array}$ & $\begin{array}{l}129068 \\
(4.9)\end{array}$ & $\begin{array}{l}15978 \\
(4.9)\end{array}$ & $<0.001$ \\
\hline Male & $44(6.0)$ & 465 (6.6) & $79(6.6)$ & $\begin{array}{l}377 \\
(8.3)\end{array}$ & $\begin{array}{l}2350 \\
(8.3)\end{array}$ & $\begin{array}{l}258 \\
(9.5)\end{array}$ & $8594(5.2)$ & $70093(5.1)$ & $8701(5.2)$ & \\
\hline Female & $49(6.9)$ & $444(6.5)$ & $51(5.0)$ & $\begin{array}{l}309 \\
(6.7)\end{array}$ & $\begin{array}{l}1991 \\
(7.3)\end{array}$ & $\begin{array}{l}170 \\
(7.0)\end{array}$ & $7235(4.5)$ & 58975 (4.6) & 7277 (4.7) & \\
\hline \multicolumn{11}{|l|}{$\begin{array}{l}\text { Year of } \\
\text { birth, } \mathrm{n}(\%)\end{array}$} \\
\hline \begin{tabular}{|l}
$2000-$ \\
2004
\end{tabular} & $\begin{array}{l}137 \\
(8.1)\end{array}$ & $\begin{array}{l}1326 \\
(78.6)\end{array}$ & $\begin{array}{l}224 \\
(13.3)\end{array}$ & $\begin{array}{l}1984 \\
(12.1)\end{array}$ & $\begin{array}{l}12924 \\
(79.1)\end{array}$ & $\begin{array}{l}1436 \\
(8.8)\end{array}$ & $\begin{array}{l}96142 \\
(9.5)\end{array}$ & $\begin{array}{l}805423 \\
(79.6)\end{array}$ & $\begin{array}{l}110562 \\
(10.9)\end{array}$ & \\
\hline $\begin{array}{l}2005- \\
2009\end{array}$ & $\begin{array}{l}442 \\
(8.8)\end{array}$ & $\begin{array}{l}3928 \\
(78.4)\end{array}$ & $\begin{array}{l}640 \\
(12.8)\end{array}$ & $\begin{array}{l}3002 \\
(13.3)\end{array}$ & $\begin{array}{l}17858 \\
(79.2)\end{array}$ & $\begin{array}{l}1683 \\
(7.5)\end{array}$ & $\begin{array}{l}104752 \\
(9.8)\end{array}$ & $\begin{array}{l}859543 \\
(80.6)\end{array}$ & $\begin{array}{l}101724 \\
(9.5)\end{array}$ & \\
\hline $\begin{array}{l}2010- \\
2015\end{array}$ & $\begin{array}{l}866 \\
(8.0)\end{array}$ & $\begin{array}{l}8581 \\
(79.4)\end{array}$ & $\begin{array}{l}1356 \\
(12.6)\end{array}$ & $\begin{array}{l}4149 \\
(13.5)\end{array}$ & $\begin{array}{l}24469 \\
(79.9)\end{array}$ & $\begin{array}{l}2005 \\
(6.5)\end{array}$ & $\begin{array}{l}126017 \\
(10.2)\end{array}$ & $\begin{array}{l}995962 \\
(80.7)\end{array}$ & $\begin{array}{l}111463 \\
(9.0)\end{array}$ & \\
\hline $\begin{array}{l}\text { Offspring } \\
\text { male sex, } \mathrm{n} \\
(\%)\end{array}$ & $\begin{array}{l}732 \\
(50.7)\end{array}$ & $\begin{array}{l}7051 \\
(51.0)\end{array}$ & $\begin{array}{l}1190 \\
(53.6)\end{array}$ & $\begin{array}{l}4537 \\
(49.7)\end{array}$ & $\begin{array}{l}28154 \\
(51.0)\end{array}$ & $\begin{array}{l}2708 \\
(52.8)\end{array}$ & $\begin{array}{l}166498 \\
(50.9)\end{array}$ & $\begin{array}{l}1368 \\
(51.4)\end{array}$ & $\begin{array}{l}167559 \\
(51.8)\end{array}$ & $<0.001$ \\
\hline
\end{tabular}

HDP, hypertensive disorders in pregnancy; NA, not applicable; SET, single embryo transfer.

a Chi-square test, Student's $t$-test.

b Data on education are not available for Norway.

c Data on paternal age are not available for Denmark.

d Data on diabetes included any diabetes due to different registration practices in the study countries. 


\section{Ethical approval}

Approvals for data retrieval and linkage were obtained in each country (Opdahl et al., 2019). In Denmark, ethical approval is not required for scientific projects solely based on register data. In Norway, ethical approval was given by the Regional Committee for Medical and Health Research Ethics (REK-Nord, 2010/1909). In Sweden, approval was obtained from the Ethical committee in Gothenburg, Dnr 214-12, T422-12, T516-15, T233-16, T300-17, T1144-17 and T121-18 and the Swedish Ethical Review Authority 2019-02347.

In addition, data retrieval for CoNARTaS was approved by the register-keeping authorities in each country (Denmark: Region H 22-06-2016, DT-journal number 2012-58-0004, local journal number AHH-2016-033, I-suite number: 04790; Norway: Norwegian Institute of Public Health, Statistics Norway, Norwegian Directorate of Health and the Cancer Registry of Norway; Sweden: National Board of Health and Welfare, Statistics Sweden) and several National Quality Registries for additional data on morbidity.

\section{Outcomes and statistical analyses}

Mean birth weights and the percentages of SGA, AGA and LGA for each GW at birth were compared between singletons born after FET, fresh ET and NC, stratified by offspring sex. Chi-square test and test for relative proportions were used to compare categorical variables and Student's $t$-test to compare continuous variables. Adjusted odds ratios (aORs) for LGA and SGA with 95\% CIs were calculated using logistic regressions first for the whole population and next categorized into extremely preterm (GW 22-27), very preterm (GW 28-31), moderate/late preterm (GW 32-36), term (GW 37-41) and post-term (GW 42-45) subgroups. Adjustments were made for year of birth, maternal age, BMI, parity, smoking, any diabetes mellitus, chronic hypertension and offspring sex. A subanalysis was performed for the moderate/late preterm, term and post-term subgroups as well as the whole population adjusting also for embryo culture time.

Sensitivity analyses were performed excluding induced births (Model 1) and excluding both induced births and births by caesarean section (Model 2) from the analyses.

All statistical analyses were performed using SAS statistical software, version 9.4. (SAS Institute).

\section{Results}

\section{Background factors}

A description of the study population is presented in Table I. There were statistically significant differences between FET and fresh ET groups for all background factors except for maternal BMI. The incidence of hypertensive disorders in pregnancy was $7.5 \%$ among FET, 5.9\% among fresh ET and $4.4 \%$ among NC $(P<0.001$ for FET versus fresh ET).

\section{Term and preterm births and birth weights}

The largest proportions of preterm (GW 22-36) births were seen among children born after fresh ET (8.4\% for boys and $7.2 \%$ for girls). The corresponding proportions among children born after FET were $6.6 \%$ for boys and $6.4 \%$ for girls. In the NC group, the proportions were $5.1 \%$ for boys and $4.6 \%$ for girls $(P<0.001$ for FET versus fresh ET) (Table I).

Mean birth weights were significantly higher for boys born after FET starting from GW 33 (range from $75 \mathrm{~g}$ to $228 \mathrm{~g}$ by week), and for girls born after FET starting from GW 34 (range from $90 \mathrm{~g}$ to $236 \mathrm{~g}$ by week), compared to fresh ET (Fig. 1a and b).

Figure 1. Mean birth weights for each gestational week for children born after frozen embryo transfer (FET), fresh embryo transfer (fresh ET) and natural conception (NC) stratified by sex of child. (a) Mean weights, boys. (b) Mean weights, girls. 


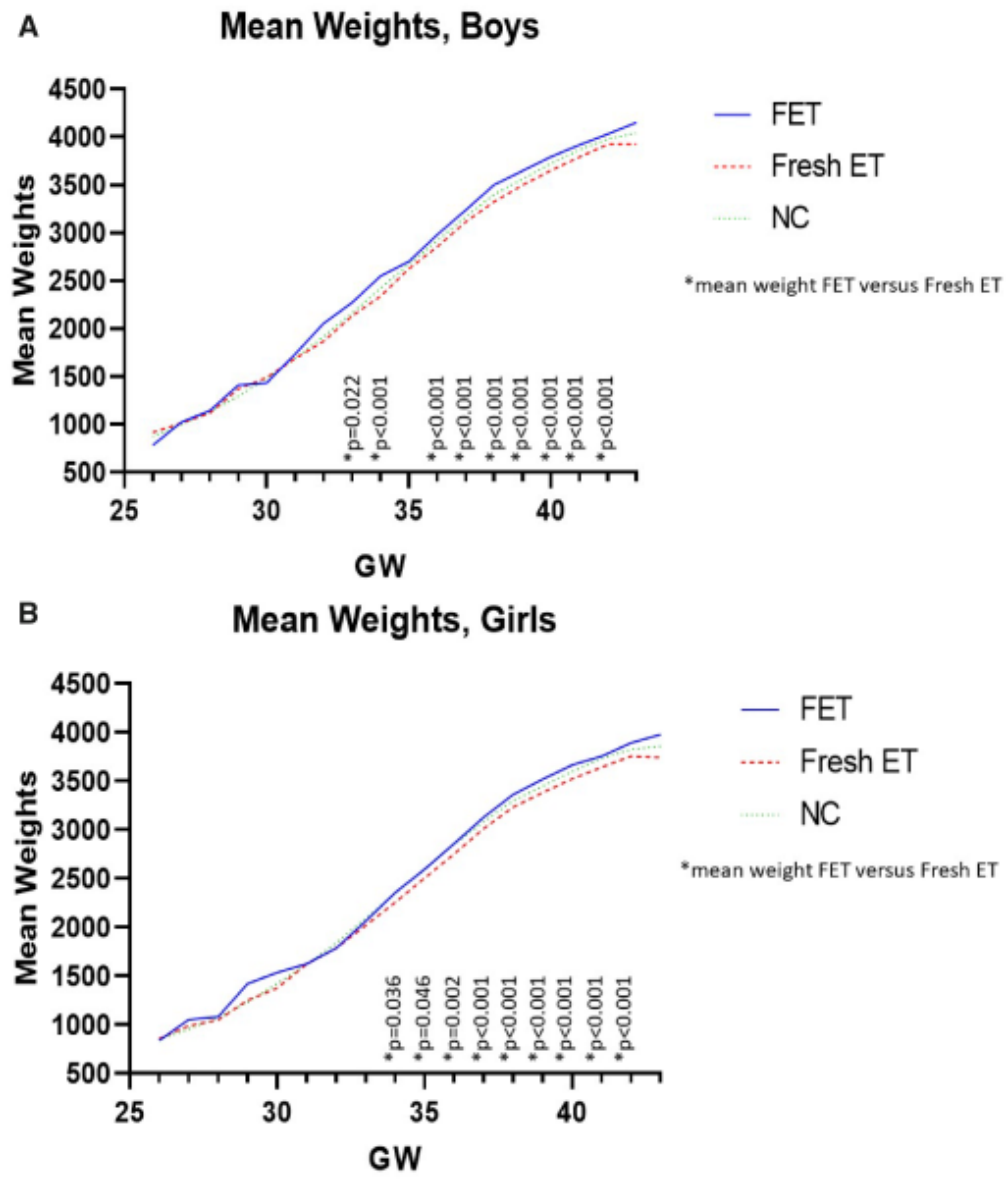

\section{Proportions of LGA ( $>90$ percentiles) and SGA ( $<10$ percentiles)}

Boys born after FET had significantly higher proportions (11.0-15.1\%) of LGA at birth compared to those born after fresh ET (7.1-9.4\%) between GW 36 and 42 (range from $P<0.001$ to $P=0.048$ by week). Girls born after FET had significantly higher proportions of LGA (10.6-13.4\%) compared to those born after fresh ET (6.6-8.0\%) between GW 37 and 42 (range from $P<0.001$ to $P=0.009$ by week). Compared to children born after NC, significantly more LGA $(12.3-15.1 \%)$ was seen for boys born after FET between GW 38 and $41(P<0.001)$ and for girls born after FET (12.6-13.4\%) between GW 37 and 40 (range from $P<0.001$ to $P=0.018$ by week) (Table II).

Table II Proportions of large-for-gestational-age (LGA, $>90$ percentiles) birth weights for boys and girls for each gestational week (GW) among frozen embryo transfer (FET), fresh embryo transfer (fresh ET) and naturally conceived (NC) pregnancies.

\begin{tabular}{|c|c|c|c|c|c|c|c|c|}
\hline & \multicolumn{4}{|l|}{ Boys } & \multicolumn{4}{|l|}{ Girls } \\
\hline & $\begin{array}{l}\text { LGA FET } \\
\mathrm{n}=1190\end{array}$ & $\begin{array}{l}\text { LGA fresh } \\
\text { ET } n= \\
2708\end{array}$ & $\begin{array}{l}\text { LGA NC n= } \\
167559\end{array}$ & $P$-value & $\begin{array}{l}\text { LGA FET } \\
\mathrm{n}=1030\end{array}$ & $\begin{array}{l}\text { LGA fresh } \\
\text { ET } n= \\
2416\end{array}$ & $\begin{array}{l}\text { LGA NC n= } \\
156190\end{array}$ & $P$-value ${ }^{\mathrm{a}}$ \\
\hline GW & n (\%) & n $(\%)$ & n (\%) & & n (\%) & n $(\%)$ & n (\%) & \\
\hline $22-25$ & $<5$ & 9 & 181 & $N A$ & $<5$ & $<5$ & 154 & $N A$ \\
\hline 26 & $-(0)$ & 7 (17.9) & $90(9.8)$ & $0.169^{\mathrm{b} ; 0.324 \mathrm{c}}$ & $<5(14.3)$ & $<5(9.8)$ & $78(9.7)$ & $0.717^{\mathrm{b} ; 0.681 \mathrm{c}}$ \\
\hline 27 & $-(0)$ & 7 (14.9) & $110(9.5)$ & $0.274^{\mathrm{b} ; 0.390 \mathrm{c}}$ & $-(0)$ & $<5(9.5)$ & $81(8.8)$ & $0.310^{\mathrm{b} ; 0.327 \mathrm{c}}$ \\
\hline
\end{tabular}




\begin{tabular}{|c|c|c|c|c|c|c|c|c|}
\hline & Boys & & & & Girls & & & \\
\hline & $\begin{array}{l}\text { LGA FET } \\
\mathrm{n}=1190\end{array}$ & $\begin{array}{l}\text { LGA fresh } \\
\text { ET } n= \\
2708\end{array}$ & $\begin{array}{l}\text { LGA NC } \mathrm{n}= \\
167559\end{array}$ & $P$-value ${ }^{\mathrm{a}}$ & $\begin{array}{l}\text { LGA FET } \\
\mathrm{n}=1030\end{array}$ & $\begin{array}{l}\text { LGA fresh } \\
\text { ET } n= \\
2416\end{array}$ & $\begin{array}{l}\text { LGA NC } \mathrm{n}= \\
156190\end{array}$ & $P$-value \\
\hline GW & n (\%) & n (\%) & n (\%) & & n (\%) & n (\%) & n (\%) & \\
\hline 28 & $<5(8.3)$ & $7(9.0)$ & $124(9.2)$ & $0.932^{\mathrm{b} ; 0.918 \mathrm{c}}$ & $-(0)$ & $<5(7.7)$ & $109(9.7)$ & $0.417^{b} ; 0.355 \mathrm{c}$ \\
\hline 29 & $<5(28.6)$ & $9(12.9)$ & $156(9.1)$ & $0.138^{\mathrm{b} ;} \boldsymbol{0 . 0 1 2 ^ { \mathrm { c } }}$ & $<5(16.7)$ & $7(11.3)$ & $131(9.8)$ & $0.602^{\mathrm{b} ; 0.426 \mathrm{c}}$ \\
\hline 30 & $<5(5.6)$ & $11(9.3)$ & $216(9.9)$ & $0.600^{\mathrm{b} ; 0.539 \mathrm{c}}$ & $<5(22.2)$ & $7(8.3)$ & $174(9.9)$ & $0.085^{\mathrm{b} ; 0.083 \mathrm{c}}$ \\
\hline 31 & $<5(9.1)$ & $8(7.2)$ & $297(10)$ & $0.760^{\mathrm{b} ; 0.882 \mathrm{c}}$ & $<5(5.3)$ & $5(6.3)$ & $155(6.6)$ & $0.871^{\mathrm{b} ; 0.819 \mathrm{c}}$ \\
\hline 32 & $5(22.7)$ & $15(8.4)$ & $429(10)$ & $0.034^{\mathrm{b} ;} 0.047^{\mathrm{c}}$ & $-(0)$ & $8(6.0)$ & $348(10.0)$ & $0.229^{\mathrm{b} ; 0.110 \mathrm{c}}$ \\
\hline 33 & $6(12.0)$ & $23(9.8)$ & $660(10)$ & $0.638^{\mathrm{b} ; 0.637 \mathrm{c}}$ & $5(11.4)$ & $15(7.0)$ & $527(10.1)$ & $0.325^{\mathrm{b} ; 0.783 \mathrm{c}}$ \\
\hline 34 & $13(19.4)$ & $19(4.9)$ & $1133(10.1)$ & $<0.001^{\mathrm{b} ;} 0.012^{\mathrm{c}}$ & $7(10.6)$ & $24(7.7)$ & $911(10.0)$ & $0.433^{\mathrm{b} ; 0.876 \mathrm{c}}$ \\
\hline 35 & $11(9.1)$ & $46(7.9)$ & $1817(10)$ & $0.671^{\mathrm{b} ; 0.745 \mathrm{c}}$ & $10(8.8)$ & $31(6.1)$ & $1547(10.1)$ & $0.277^{\mathrm{b} ; 0.654 \mathrm{c}}$ \\
\hline 36 & $33(14.9)$ & $97(9.4)$ & $3488(10)$ & $0.015^{\mathrm{b}, \mathrm{c}}$ & $19(9.5)$ & $57(6.7)$ & $3062(10.0)$ & $0.166^{\mathrm{b} ; 0.783 \mathrm{c}}$ \\
\hline 37 & $61(12.4)$ & $176(8.3)$ & $8135(10)$ & $0.004^{b} ; 0.067^{c}$ & 57 (13.4) & $152(7.7)$ & $7650(10.0)$ & $<0.001^{\mathrm{b} ;} 0.018^{\mathrm{c}}$ \\
\hline 38 & $193(15.1)$ & $365(7.1)$ & $21879(9.9)$ & $<0.001^{b, c}$ & $148(12.6)$ & $388(7.8)$ & $21583(9.8)$ & $<0.001^{\mathrm{b} ;} 0.002^{\mathrm{c}}$ \\
\hline 39 & $269(15.0)$ & 584 (7.6) & 37612 (9.9) & $<0.001^{\mathrm{b}, \mathrm{c}}$ & $229(12.9)$ & $512(6.6)$ & $34606(9.0)$ & $<0.001^{\mathrm{b}, \mathrm{c}}$ \\
\hline 40 & 277 (12.3) & $692(7.6)$ & $45662(9.7)$ & $<0.001^{\mathrm{b}, \mathrm{c}}$ & $278(12.8)$ & $636(6.9)$ & 46539 (10.0) & $<0.001^{b, c}$ \\
\hline 41 & $239(12.5)$ & $474(7.4)$ & $33167(9.9)$ & $<0.001^{b, c}$ & 195 (10.6) & $433(7.0)$ & $29564(10.0)$ & $<\mathbf{0 . 0 0 1}^{\mathrm{b} ; 0.340 \mathrm{c}}$ \\
\hline 42 & $71(11.0)$ & $154(8.4)$ & $11961(10)$ & 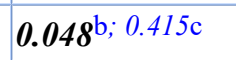 & $68(11.6)$ & $122(8.0)$ & $8647(9.9)$ & $0.009 \mathrm{~b} ; 0.150 \mathrm{c}$ \\
\hline $43-44$ & & 5 & 442 & NA & $<5$ & $<5$ & 324 & $N A$ \\
\hline
\end{tabular}

NA, not applicable. Bold indicates statistical significance.

a Test for relative proportions.

b FET versus fresh ET.

c FET versus NC.

The proportion of SGA was significantly lower among boys born after FET (7.6-8.7\%) compared to those born after fresh ET (11.9-13.6\%) between GW 36 and 42 (range from $P<0.001$ to $P=0.016$ by week). For girls born after FET, the difference was seen between GW 38 and 42 (7.0-9.3\%) compared to those born after fresh ET (13.0-14.6\%) $(P<0.001)$. Compared to children born after NC, significantly more SGA was seen for boys born after fresh ET (11.9-13.4\%) between GW 38 and 39 (range from $P=0.002$ to $P=0.015$ by week) and for girls born after fresh ET (13.2-13.6\%) between GW 38 and 40 (range from $P=0.003$ to $P=0.015$ by week) (data not shown).

\section{Adjusted odds ratios for LGA ( $>90$ percentiles) and SGA ( $<10$ percentiles)}

All singletons born after FET had a significantly greater risk of being LGA (crude odds ratio (OR) $1.82,95 \% \mathrm{CI}$ 1.73-1.92; aOR 1.87, 95\% CI 1.76-1.98) compared to fresh ET. When gestational age at birth was categorized into extremely preterm (GW 22-27), very preterm (GW 28-31), moderate/late preterm (GW 32-36), term (GW 37-41) and post-term (GW 42-45) subgroups, statistically significant differences in the aOR for LGA were found for moderate/late preterm (aOR 1.84, 95\% CI 1.40-2.40), term (aOR 1.91, 95\% CI 1.79-2.04) and post-term (aOR 1.53, 95\% CI 1.20-1.96) FET versus fresh ET subgroups (Table III). Adjusting for embryo culture time did not change the results (data not shown).

Table III Crude odds ratio (OR) and adjusted odds ratio (aOR) for large-for-gestational-age (LGA, >90 percentiles) categorized by gestational age for frozen embryo transfer (FET) versus fresh embryo transfer (fresh ET). 


\begin{tabular}{|l|l|l|l|l|}
\hline \multicolumn{5}{|c|}{ FET versus Fresh ET } \\
\hline GW & \multicolumn{1}{|l}{ Crude OR $95 \%$ CI } & aOR $95 \%$ CI \\
\hline $22-27$ & 0.77 & $0.31-1.90$ & 0.32 & $0.07-1.41$ \\
\hline $28-31$ & 1.45 & $0.79-2.65$ & 1.39 & $0.66-2.92$ \\
\hline $32-36$ & $\mathbf{1 . 6 2}$ & $\mathbf{1 . 2 9}-\mathbf{2 . 0 4}$ & $\mathbf{1 . 8 4}$ & $\mathbf{1 . 4 0}-\mathbf{2 . 4 0}$ \\
\hline $37-41$ & $\mathbf{1 . 8 8}$ & $\mathbf{1 . 7 8}-\mathbf{1 . 9 9}$ & $\mathbf{1 . 9 1}$ & $\mathbf{1 . 7 9}-\mathbf{2 . 0 4}$ \\
\hline $42-45$ & $\mathbf{1 . 3 9}$ & $\mathbf{1 . 1 3}-\mathbf{1 . 7 2}$ & $\mathbf{1 . 5 3}$ & $\mathbf{1 . 2 0}-\mathbf{1 . 9 6}$ \\
\hline All & $\mathbf{1 . 8 2}$ & $\mathbf{1 . 7 3}-\mathbf{1 . 9 2}$ & $\mathbf{1 . 8 7}$ & $\mathbf{1 . 7 6}-\mathbf{1 . 9 8}$ \\
\hline
\end{tabular}

Logistic regression, crude and adjusted OR (year of birth, maternal age, parity, BMI, chronic hypertension, diabetes, smoking and sex of child). Bold indicates statistical significance.

GW, gestational week.

When stratified by sex, the risks of LGA associated with FET were similar for boys (crude OR 1.84, 95\% CI 1.711.98; aOR 1.84, 95\% CI 1.69-2.01) and girls (crude OR 1.80, 95\% CI 1.66-1.94; aOR 1.90, 95\% CI 1.73-2.07) compared to fresh ET (data not shown).

Also compared to NC, the risk of LGA was greater among children born after FET (crude OR 1.34, 95\% CI 1.281.40; aOR 1.28, 95\% CI 1.22-1.35). Categorized by gestational age at birth, significant differences were found for moderate/late preterm (aOR 1.37, 95\% CI 1.09-1.74) and term (aOR 1.30, 95\% CI 1.23-1.37) FET versus NC subgroups (Table IV).

Table IV Crude odds ratio (OR) and adjusted odds ratio (aOR) for large-for-gestational-age (LGA, >90 percentiles) categorized by gestational age for frozen embryo transfer (FET) versus natural conception (NC).

\begin{tabular}{|l|l|l|l|l|}
\hline \multicolumn{5}{|l|}{ FET versus NC } \\
\hline GW & Crude OR 95\% CI & \multicolumn{2}{l|}{ aOR 95\% CI } \\
\hline $22-27$ & 0.76 & $0.33-1.74$ & 0.41 & $0.10-1.67$ \\
\hline $28-31$ & 1.37 & $0.80-2.36$ & 1.56 & $0.82-2.95$ \\
\hline $32-36$ & 1.19 & $0.98-1.46$ & $\mathbf{1 . 3 7}$ & $\mathbf{1 . 0 9}-\mathbf{1 . 7 4}$ \\
\hline $37-41$ & $\mathbf{1 . 3 7}$ & $\mathbf{1 . 3 1}-\mathbf{1 . 4 4}$ & $\mathbf{1 . 3 0}$ & $\mathbf{1 . 2 3}-\mathbf{1 . 3 7}$ \\
\hline $42-45$ & 1.17 & $0.98-1.39$ & 1.16 & $0.96-1.41$ \\
\hline All & $\mathbf{1 . 3 4}$ & $\mathbf{1 . 2 8}-\mathbf{1 . 4 0}$ & $\mathbf{1 . 2 8}$ & $\mathbf{1 . 2 2}-\mathbf{1 . 3 5}$ \\
\hline
\end{tabular}

Logistic regression, crude and adjusted OR (year of birth, maternal age, parity, BMI, chronic hypertension, diabetes, smoking and sex of child). Bold indicates statistical significance.

GW, gestational week.

All singletons born after FET had a significantly lower risk of being SGA (crude OR 0.60, 95\% CI 0.56-0.63; aOR $0.61,95 \%$ CI $0.57-0.65$ ) compared to fresh ET. Also compared to NC, singletons born after FET had a slightly lower risk of being SGA (OR 0.82, 95\% CI 0.78-0.87; aOR 0.91, 95\% CI 0.85-0.96) (data not shown).

\section{Sensitivity analyses}

To test the robustness of the findings, we performed sensitivity analyses first excluding induced births (Model 1) and next excluding both induced births and births by caesarean section (Model 2), mainly to exclude cases with iatrogenic preterm or term birth (e.g. induced birth because of pregnancy complications). There were 4446 inductions and 5016 caesarean sections in the FET group. The respective numbers for fresh ET were 13726 and 17685 (Table I). The final group sizes in Model 2 were 9309 for FET, 41544 for fresh ET and 2362812 for NC. The differences between FET and fresh ET remained unchanged in the moderate/late preterm, term and post-term groups, and be- 
tween FET and NC in the moderate/late preterm and term groups. For all FET versus fresh ET, the aOR for LGA was 1.90, 95\% CI 1.77-2.04 in Model 1. In Model 2, aOR for FET versus fresh ET was 1.89, 95\% CI 1.73-2.07. For FET versus NC, the aOR for LGA was 1.31, 95\% CI 1.23-1.38 in Model 1 and 1.22, 95\% CI 1.13-1.31 in Model 2.

\section{Discussion}

The main finding in this large Nordic population-based cohort study is that the differences in birth weight between children born after FET and fresh ET become apparent and statistically significant in the last trimester of pregnancy from GW 33 onwards when comparing mean birth weights. Considering the risk of LGA, the differences show later on, starting from GW 36, becoming significant slightly earlier for boys than girls.

To date, our study is the largest study on birth weights among preterm and term ART singletons with an NC control group. The population-based design adds to the reliability of these results. In addition, the Nordic registers have high coverage, and linking of information is possible because of the individual national identity numbers (Opdahl et al., 2019).

As a limitation to our study, there lies a possible bias in using birth weight as the study outcome: LGA or other growth deviations will only be picked up among the children who are born at a certain GW, not among those who carry on growing in utero. Several causes of spontaneous preterm birth or iatrogenic preterm delivery may influence foetal growth and as such, birth weight. Unfortunately, we had no information on the reasons for preterm births in our study. In the sensitivity analyses, we aimed to test our results excluding induced births and births by caesarean section, thus excluding iatrogenic or spontaneous births caused by severe pregnancy complications and found that the results remain similar. Another limitation is that, within this study design, we have no information on the growth between implantation and GW 22. Considering the time span and different registration practices in the four Nordic countries, unfortunately, the CoNARTaS data do not include information on the embryo culture media that have been used. It also needs to be taken into account that even in a large study cohort, the number of children born in the extremely preterm or post-term weeks is quite low. As an observational cohort, there may also be residual confounding that we could not control for. However, the study design that we are presenting is the easiest way to look at this phenomenon in a large population as foetal ultrasound weight estimate comparison is prone to error (Salomon et al., 2019) and also laborious to carry out.

Previously, birth weights of preterm and term ART children have been described in an Australian cohort where birthweight for gestational age percentile charts were developed for singleton births following ART treatment. Previously published general population growth charts acted as reference in this study of about 70000 live born ART singletons from years 2002 to 2010 . The proportion of LGA ( $>90$ percentiles) for boys born after FET was significantly higher than that of the general population between GW 33 and 41. For girls born after FET, this difference was seen between GW 34 and 42. Boys and girls born after fresh ET had significantly more SGA ( $<10$ percentiles) at GW 4142 and 40-41, respectively (Li et al., 2014). Compared to our study, the risk of LGA associated with FET compared to general population became statistically significant earlier in the pregnancy. In the study by Li et al. (2014), FET was not compared to fresh ET, and no crude or background-aORs for LGA or SGA were calculated.

In a recent French retrospective cohort of 560 singleton pregnancies, foetal ultrasound growth estimates were compared to reference curves in each trimester. Four groups were compared: pregnancies originated from IVF, ICSI, FET and IUI. At the first (GW 11-13) and second (GW 21-23) trimester US examinations, the estimated foetal sizes (mean $z$ scores) for all groups were significantly larger than the 0 SD reference curve. At the third trimester US examination (GW 31-33), foetal growth had slowed down for the IVF, ICSI and IUI groups, but not for FET foetuses, which remained significantly larger than the 0 SD reference curve (Ginod et al., 2018). This difference in the growth between FET and fresh ET (IVF and ICSI) developing by the last trimester seems compatible with our results.

In a previous study by the CoNARTaS group, the aOR of LGA was 1.45 (95\% CI 1.27-1.64) for children born after FET compared to fresh ET (Wennerholm et al., 2013). It should be noted that LGA was classified as birth weight $\geq 2$ SD and adjustments were made for maternal age, parity, year of birth, offspring sex and country of origin. In our study, a larger and more recent (but partly overlapping) study population was studied, with a less restrictive definition of LGA (birth weight $\geq 90$ percentiles), and the aOR for LGA was 1.87, 95\% CI 1.76-1.98 (adjusted for year of birth, maternal age, parity, BMI, chronic hypertension, diabetes, smoking and offspring sex) for singletons born after FET compared to fresh ET. During the study period of Wennerholm et al. (2013) mostly cleavage stage slow-frozen embryos were used in FET, which in recent years has been replaced with more vitrification and blasto- 
cyst transfer. It has been suggested that extended embryo culture time might be associated with higher birthweights (Zhu et al., 2014; Martins et al., 2016; Zhang et al., 2019). In a subanalysis of our study cohort, adjusting for embryo culture time did not significantly change the results on the risk of LGA. Furthermore, in a recent CoNARTaS study by Ginström Ernstad et al. (2019a), no difference was found in the risk of LGA between children born after vitrified blastocyst transfer and slow-frozen cleavage stage embryo transfer. Since vitrification was introduced simultaneously with blastocyst transfers, it was not possible to investigate the impact of freezing method and culture time separately (Ginström Ernstad et al., 2019a). In a previous Finnish study, the freezing method did not impact the risk of LGA (Kaartinen et al., 2016).

There is a wide physiological range in human foetal growth (Gardosi et al., 2018). Male foetus, advanced maternal age, height, weight and parity all have a positive effect on foetal growth estimated from ultrasound measurement. Also, ethnicity has a substancial role on foetal growth (Kiserud et al., 2017). In our study, we were able to control for offspring sex, maternal age, BMI and parity, and also smoking, chronic hypertension and diabetes. Information on ethnicity was not available, but since the majority of the Nordic population is of Caucasian origin, this is not likely to be a major confounding factor in our study. Unfortunately, we had no information on gestational diabetes. Our data on diabetes included any diabetes due to different registration practices in the study countries. However, in the metaanalysis by Sha et al. (2018), no difference in the amount of gestational diabetes was shown between pregnancies originating after FET and fresh ET.

Several factors may contribute to foetal growth differences between children born after FET, fresh ET and NC. The underlying cause for infertility/subfertility of the parents, laboratory procedures, embryo culture medium, culture length and patient or embryo selection through the freeze-thaw process may have an influence. Placentation may be altered due to the supraphysiological blood concentrations of oestradiol and progesterone following ovarian stimulation and fresh ET, lasting at least through the first trimester (Järvelä et al., 2014) and possibly causing increased risk of low birth weight (Pereira et al., 2017). Varying epigenetic changes and differences in gene expression in placentas and children from pregnancies originating after FET, fresh ET and NC have been discovered (Estill et al., 2016; Choux et al., 2018; Lee et al., 2019). It has also been suggested that the effect of ART on DNA methylation in placenta is genotype specific (Marjonen et al., 2018).

The consequences of these changes on the long-term health of the ART offspring are still largely unclear. It is known that the perinatal health of children originating after ART has improved in the past years, greatly explained by the reduced amount of multiple gestations after introduction of FET and eSET (Henningsen et al., 2015). It is noteworthy, that the risk of LGA is pronounced in FET pregnancies, even as the risk of hypertensive disorders is also higher compared to fresh ET and NC pregnancies (Opdahl et al., 2015; Maheshwari et al., 2018; Sha et al., 2018). The absence of a corpus luteum in programmed FET cycles has been shown to be associated with higher risk of hypertensive disorders in pregnancy and neonatal macrosomia (birth weight $>4500 \mathrm{~g}$ ) compared to FET in natural or stimulated cycles (Ginström Ernstad et al., 2019b). In our study, we unfortunately had no data on the different FET cycle modalities.

A question has been raised about whether the effects of the freeze-thaw process depend on the offspring sex. In our study, we found the weight difference to become statistically significant slightly earlier in boys than girls, in accordance with Li et al. (2014). The observed gender difference may, however, also be caused by statistical power, since there are more boys than girls among preterm births. In a study by Kaartinen et al. (2015), male sex was found to be more affected by extended embryo culture times as regards to LGA birth weight. In a recent single-centre study of 1295 singletons, term boys born after FET from blastocyst transfers in artificial cycles were found to have significantly greater risk of LGA compared to girls (Coetzee et al., 2020), and in a nationwide cohort from the US, consisting of 180184 singletons (Litzky et al., 2018), FET had a slightly stronger association with larger birthweights of term boys compared to girls. Our results somewhat contradict these findings, as there were no sex-dependent differences in the aOR for LGA in the whole study population or in the term-born subgroup.

It is unlikely that small differences in birth weight should cause any problems for the offspring. In addition, there is evidence that the ponderal index of FET newborns is in line with those of fresh ET and NC newborns, suggesting that FET children are not 'fat', just large in size (Pelkonen et al., 2010). Still, concerning LGA birthweight, clear consequences have been shown. LGA at term is associated with certain perinatal risks for both the mother and the neonate, such as birth trauma (Khambalia et al., 2017; Doty et al., 2019). A recent study also suggests that pregnan- 
cies complicated by LGA are at increased risk of stillbirth at or beyond GW 36, independent of maternal diabetic status (Carter et al., 2019). On the other hand, extremely or very premature LGA infants (GW 22-29) had better inhospital outcomes compared to AGA infants in a recently published retrospective cohort (Boghossian et al., 2018). Children who are born LGA have been shown to have a higher risk for obesity later in childhood, school-age and adulthood (Taal et al., 2013; Kapral et al., 2018; Derraik et al., 2020), which may lead to elevated cardiometabolic risks later in life.

In conclusion, the freeze-thaw process seems to be associated with excess foetal growth, leading to higher birthweights and greater risk of LGA at least in the last trimester of pregnancy. This is a major aspect of the safety profile of ART treatments. Our results add to the understanding of the timing of these differences. While FET and eSET have been important steps forward in reducing the risks associated with multiple gestations, more research is needed on the possible risks associated with LGA birth weights, especially combined with preterm birth and long-term outcomes of these ART children.

\section{Data availability}

The Nordic register keepers have given permission for this specific study, and the data cannot be shared without authorization from the register keepers. Interested research collaborators can contact the CoNARTaS researchers at www.conartas.com.

Acknowledgements

We wish to thank Professor Hannu Martikainen, Oulu University, Finland, for his contribution in the original idea of the study design. We also warmly thank the Nordic fertility clinics for their meticulous reporting of the ART data throughout the years.

\section{Authors' roles}

All authors contributed to the obtaining and preparing the data. A.M.T., S.P., A.T. and M.G. participated in the study design. M.G. analysed the data. A.M.T., S.P., M.G. and A.T. drafted the manuscript. All authors contributed in the revisions of the manuscript and approved the final version.

\section{Funding}

The CoNARTaS collaboration has received the following funding: the Nordic Trial Alliance: a pilot project jointly funded by the Nordic Council of Ministers and NordForsk [71450], the Central Norway Regional Health Authorities [46045000], the Norwegian Cancer Society [182356-2016], the Nordic Federation of Obstetrics and Gynaecology [NF13041, NF15058, NF16026 and NF17043], the Interreg Öresund-Kattegat-Skagerrak European Regional Development Fund (ReproUnion project) and the Research Council of Norway's Centre of Excellence funding scheme [262700]. The funding sources had no role in designing or conducting the study or in the decision to submit the manuscript for publication. [AQ4]

\section{Conflict of interest}

None reported.

\section{References [AQ5]}

Barker DJ. The origins of the developmental origins theory. J Intern Med 2007;261:412-417.

Boghossian NS, Geraci M, Edwards EM, Horbar JD. In-hospital outcomes in large for gestational age infants at 22-29 weeks of gestation. J Pediatr 2018;198:174-180.

Carter EB, Stockburger J, Tuuli MG, Macones GA, Odibo AO, Trudell AS. Large-for-gestational-age and stillbirth: is there a role for antenatal testing? Ultrasound Obstet Gynecol 2019;54:334-337.

Choux C, Binquet C, Carmignac V, Bruno C, Chapusot C, Barberet J, Lamotte M, Sagot P, Bourc'his D, Fauque P. The epigenetic control of transposable elements and imprinted genes in newborns is affected by the mode of conception: ART versus spontaneous conception without underlying infertility. Hum Reprod 2018;33:331-340. 
Coetzee K, Ozgur K, Bulut H, Berkkanoglu M, Humaidan P. Large-for-gestational-age is male-gender dependent in artificial frozen embryo transfers cycles: a cohort study of 1295 singleton live births. Reprod Biomed Online 2020;40:134-141.

De Geyter C, Calhaz-Jorge C, Kupka MS, Wyns C, Mocanu E, Motrenko T, Scaravelli G, Smeenk J, Vidakovic S, Goossens V; European IVF-monitoring Consortium (EIM) for the European Society of Human Reproduction and Embryology (ESHRE). ART in Europe, 2015: results generated from European registries by ESHRE. Hum Reprod Open 2020;2020:hoz038.

Derraik JGB, Maessen SE, Gibbins JD, Cutfield WS, Lundgren M, Ahlsson F. Large-for-gestational-age phenotypes and obesity risk in adulthood: a study of 195,936 women. Sci Rep 2020;10:2157.

Doty MS, Chen HY, Sibai BM, Chauhan SP. Maternal and neonatal morbidity associated with early term delivery of large-for-gestational-age but nonmacrosomic neonates. Obstet Gynecol 2019;133:1160-1166.

Estill MS, Bolnick JM, Waterland RA, Bolnick AD, Diamond MP, Krawetz SA. Assisted reproductive technology alters deoxyribonucleic acid methylation profiles in bloodspots of newborn infants. Fertil Steril 2016;106:629-639.

Gardosi J, Francis A, Turner S, Williams M. Customized growth charts: rationale, validation and clinical benefits. Am J Obstet Gynecol 2018;218:S609-S618.

Ginod P, Choux C, Barberet J, Rousseau T, Bruno C, Khallouk B, Sagot P, Astruc K, Fauque P. Singleton fetal growth kinetics depend on the mode of conception. Fertil Steril 2018;110:1109-1117.e2.

Ginström Ernstad E, Spangmose AL, Opdahl S, Henningsen AA, Romundstad LB, Tiitinen A, Gissler M, Wennerholm UB, Pinborg A, Bergh C et al. Perinatal and maternal outcome after vitrification of blastocysts: a Nordic study in singletons from the CoNARTaS group. Hum Reprod 2019a;34:2282-2289.

Ginström Ernstad E, Wennerholm UB, Khatibi A, Petzold M, Bergh C. Neonatal and maternal outcome after frozen embryo transfer: Increased risks in programmed cycles. Am J Obstet Gynecol 2019b;221:126.e1-126.e18.

Henningsen AA, Gissler M, Skjaerven R, Bergh C, Tiitinen A, Romundstad LB, Wennerholm UB, Lidegaard O, Nyboe Andersen A, Forman JL et al. Trends in perinatal health after assisted reproduction: a Nordic study from the CoNARTaS group. Hum Reprod 2015;30:710-716.

Järvelä IY, Pelkonen S, Uimari O, Mäkikallio K, Puukka K, Ruokonen A, Tekay A, Martikainen H. Contolled ovarian hyperstimulation leads to high progesterone and estradiol levels during early pregnancy. Hum Reprod 2014;29:23932401.

Kaartinen N, Kananen K, Huhtala H, Keränen S, Tinkanen H. The freezing method of cleavage stage embryos has no impact on the weight of the newborns. J Assist Reprod Genet 2016;33:393-399.

Kaartinen NM, Kananen KM, Rodriguez-Wallberg KA, Tomás CM, Huhtala HS, Tinkanen HI. Male gender explains increased birthweight in children born after transfer of blastocysts. Hum Reprod 2015;30:2312-2320.

Kapral N, Miller SE, Scharf RJ, Gurka MJ, DeBoer MD. Associations between birthweight and overweight and obesity in school-age children. Pediatr Obes 2018;13:333-341.

Khambalia AZ, Algert CS, Bowen JR, Collie RJ, Roberts CL. Long-term outcomes for large for gestational age infants born at term. J Paediatr Child Health 2017;53:876-881.

Kiserud T, Piaggio G, Carroli G, Widmer M, Carvalho J, Neerup JL, Giordano D, Cecatti JG, Abdel AH, Talegawkar SA. The World Health Organization fetal growth charts: a multinational longitudinal study of ultrasound biometric measurements and estimated fetal weight. PLoS Med 2017;14:1-36.

Lee B, Koeppel AF, Wang ET, Gonzalez TL, Sun T, Kroener L, Lin Y, Joshi NV, Ghadiali T, Turner SD et al. Differential gene expression during placentation in pregnancies conceived with different fertility treatments compared with spontaneous pregnancies. Fertil Steril 2019;111:535-546.

Li Z, Wang YA, Ledger W, Sullivan EA. Birth weight percentiles by gestational age for births following assisted reproductive technology in Australia and New Zealand, 2002-2010. Hum Reprod 2014;29:1787-1800. 
Litzky JF, Boulet SL, Esfandiari N, Zhang Y, Kissin DM, Theiler RN, Marsit CJ. Effect of frozen/thawed embryo transfer on birthweight, macrosomia, and low birthweight rates in US singleton infants. Am J Obstet Gynecol 2018;218:433.e1-433.e10.

Maheshwari A, Pandey S, Amalraj Raja E, Shetty A, Hamilton M, Bhattacharya S. Is frozen embryo transfer better for mothers and babies? Can cumulative meta-analysis provide a definitive answer? Hum Reprod Update 2018;24:35-58.

Marjonen H, Auvinen P, Kahila H, Tšuiko O, Kõks S, Tiirats A, Viltrop T, Tuuri T, Söderström-Anttila V, Suikkari $\mathrm{AM}$ et al. rs 10732516 polymorphism at the IGF2/H19 locus associates with genityope-specific effects on placental DNA methylation and birth weight of newborns conceived by assisted reproductive technology. Clin Epigenet 2018;10:80.

Marsal K, Persson PH, Larsen T, Lilja H, Selbing A, Sultan B. Intrauterine growth curves based on ultrasonically estimated foetal weights. Acta Paediatr 1996;85:834-848.

Martins WP, Nastri CO, Rienzi L, van der Poel SZ, Gracia CR, Racowsky C. Obstetrical and perinatal outcomes following blastocyst transfer compared to cleavage transfer: a systematic review and meta-analysis. Hum Reprod 2016;31:2561-2569.

Opdahl S, Henningsen AA, Bergh C, Gissler M, Romundstad LB, Petzold M, Tiitinen A, Wennerholm UB, Pinborg AB. Data resource profile: Committee of Nordic Assisted Reproductive Technology and Safety (CoNARTaS) cohort. Int J Epidemiol 2019;49:365-366f.

Opdahl S, Henningsen AA, Tiitinen A, Bergh C, Pinborg A, Romundstad PR, Wennerholm UB, Gissler M, Skjærven $\mathrm{R}$, Romundstad LB. Risk of hypertensive disorders in pregnancies following assisted reproductive technology: a cohort study from the CoNARTaS group. Hum Reprod 2015;30:1724-1731.

Pelkonen S, Koivunen R, Gissler M, Nuojua-Huttunen S, Suikkari AM, Hydén-Granskog C, Martikainen H, Tiitinen A, Hartikainen AL. Perinatal outcome of children born after frozen and fresh embryo transfer: the Finnish cohort study 1995-2006. Hum Reprod 2010;25:914-923.

Pereira N, Elias RT, Christos PJ, Petrini AC, Hancock K, Lekovich JP, Rosenwaks Z. Supraåphysiologic estradiol is an independent predictor of low birth weight in full-term singletons born after fresh embryio transfer. Hum Reprod 2017;32:1410-1417.

Pinborg A, Henningsen AA, Loft A, Malchau SS, Forman J, Andersen AN. Large baby syndrome in singletons born after frozen embryo transfer (FET): is it due to maternal factors or the cryotechnique? Hum Reprod 2014;29:618627.

Salomon LJ, Alfirevic Z, Da Silva Costa F, Deter RL, Figueras F, Ghi T, Glanc P, Khalil A, Lee W, Napolitano R et al. ISUOG Practice Guidelines: ultrasound assessment of fetal biometry and growth. Ultrasound Obstet Gynecol 2019;53:715-723.

Sazonova A, Källen K, Thurin-Kjellberg A, Wennerholm UB, Bergh C. Obstetric outcome in singletons after in vitro fertilization with cryopreserved/thawed embryos. Hum Reprod 2012;27:1343-1350.

Sha T, Yin X, Cheng W, Massey IY. Pregnancy-related complications and perinatal outcomes resulting from transfer of cryopreserved versus fresh embryos in vitro fertilization: a meta-analysis. Fertil Steril 2018;109:330-342.e9.

Taal HR, Vd Heijden AJ, Steegers EA, Hofman A, Jaddoe VW. Small and large size for gestational age at birth, infant growth, and childhood overweight. Obesity (SilverSpring). 2013;21:1261-1268.

Thurin A, Hausken J, Hillensjö T, Jablonowska B, Pinborg A, Strandell A, Bergh C. Elective single-embryo transfer versus double-embryo transfer in in vitro fertilization. N Engl J Med 2004;351:2392-2402.

Tiitinen A, Gissler M. Effect of in vitro fertilization practices on multiple pregnancy rates in Finland. Fertil Steril 2004;82:1689-1690.

Wennerholm UB, Henningsen AK, Romundstad LB, Bergh C, Pinborg A, Skjaerven R, Forman J, Gissler M, Nygren KG, Tiitinen A. Perinatal outcomes of children born after frozen-thawed embryo transfer: a Nordic cohort study from the CoNARTaS group. Hum Reprod 2013;28:2545-2553. 
Zhang J, Wang Y, Liu H, Mao X, Chen Q, Fan Y, Xiao Y, Kuang Y. Effect of in vitro culture period on birth weight after vitrified-warmed transfer cycles: analysis of 4,201 singleton newborns. Fertil Steril 2019;111:97-104.

Zhu J, Lin S, Li M, Chen L, Lian Y, Liu P, Qiao J. Effect of in vitro culture period on birthweight of singleton newborns. Hum Reprod 2014;29:448-454. 